\title{
Emphysematous pyelitis
}

\section{Chia-Chun Chiang MD, Yuh-Shiun Jong MD, Wei-Jie Wang MD}

Previously published at www.cmaj.ca

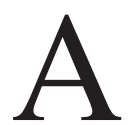

48-year-old woman presented to the emergency department with three days of fever, along with rigors, left flank pain and oliguria. She had diabetic nephropathy with a baseline serum creatinine level of 247.5 (normal < 133) $\mu \mathrm{mol} / \mathrm{L}$ and an estimated glomerular filtration rate of 19 (normal $91-130$ ) $\mathrm{mL} / \mathrm{min} / 1.73 \mathrm{~m}^{2}$. The patient's temperature was $38.8^{\circ} \mathrm{C}$, blood pressure was $139 / 73 \mathrm{~mm} \mathrm{Hg}$ and she had tenderness at the left costovertebral angle.

Laboratory studies revealed a hemoglobin concentration of 90 (normal 120-160) g/L, leukocyte count 31.48 (normal 4.3-10.8) $\times 10^{9} / \mathrm{L}$, platelet count 296 (normal 130-400) $\times$ $10 \% / \mathrm{L}$ and hemoglobin $\mathrm{A}_{1 \mathrm{C}}$ of $8.5 \%$. Serum creatinine was 530.4 (normal $<133$ ) $\mu \mathrm{mol} / \mathrm{L}$ and urinalysis showed $5-10$ white blood cells per high-power field. A plain radiograph showed a rosette-like arrangement of gas in the collecting system of the patient's left kidney and upper ureter (Figure 1A). An unenhanced computed tomography (CT) scan of the abdomen showed air in the dilated renal calyces, pelvis and upper ureter (Figure 1B). No evidence of urinary tract obstruction was found. The patient was diagnosed with emphysematous pyelitis. Peripheral blood and urine cultures grew Escherichia coli, sensitive to ceftriaxone. Treatment with nephrostomy and intravenous ceftriaxone $2 \mathrm{~g}$ daily led to clinical and radiological improvement. On follow-up at three weeks, the patient's creatinine level was $265.2 \mu \mathrm{mol} / \mathrm{L}$ and estimated glomerular filtration rate $18 \mathrm{~mL} / \mathrm{min} / 1.73 \mathrm{~m}^{2}$.

Huang and Tseng ${ }^{1}$ defined four classes of emphysematous pyelonephritis in 2000; our patient had class 1 emphysematous pyelonephritis (so-called emphysematous pyelitis). Emphysematous pyelonephritis occurs more commonly in middle-aged women with poorly controlled diabetes or urinary tract obstruction. It should be considered in patients with pyelonephritis who develop thrombocytopenia, acute renal failure, reduced level of consciousness or shock. These factors also contribute to increased mortality. ${ }^{2}$ Escherichia coli is the most common organism found in emphysematous pyelonephritis, followed by Klebsiella pneumoniae.

\footnotetext{
From the Department of Internal Medicine (Chiang, Jong, Wang) Taoyuan General Hospital, Department of Health, Executive Yuan, Taoyuan, Taiwan, and the Department of Nursing (Wang), Chang-Gung Institute of Technology, Taoyuan, Taiwan

CMAJ 2010. DOI:10.1503/cmaj.091055
}
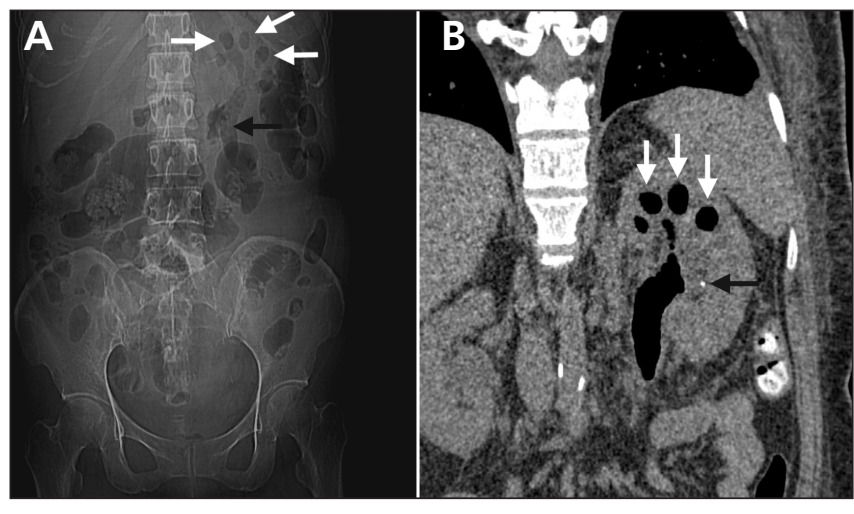

Figure 1: (A) Plain radiograph of a 48-year-old woman showing rosette-like arrangement of gas in the collecting system of her left kidney (white arrows) and upper ureter (black arrow). (B) Coronal view of an abdominal computed tomography scan showing air in the dilated renal calyces, pelvis and upper ureter (white arrows). A tiny renal stone (black arrow) is present.

Ultrasonography with plain radiography is recommended in all patients with diabetes who have acute pyelonephritis. Imaging is not generally recommended in patients who are otherwise well who respond to therapy within 72 hours. ${ }^{3}$ Computed tomography is the most sensitive method to demonstrate gas within and adjacent to the kidney. ${ }^{3}$ The initial management of emphysematous pyelonephritis includes resuscitation, antibiotic treatment targeting Gram-negative bacteria and control of diabetes if present. ${ }^{2}$ Most patients require nephrostomy. Emphysematous pyelitis has an excellent prognosis with prompt diagnosis and treatment.

This article has been peer reviewed.

Competing interests: None declared.

\section{REFERENCES}

1. Huang JJ, Tseng CC. Emphysematous pyelonephritis: clinicoradiological classification, management, prognosis, and pathogenesis. Arch Intern Med 2000;160:797805.

2. Pontin AR, Barnes RD. Current management of emphysematous pyelonephritis. Nat Rev Urol 2009;6:272-9.

3. American College of Radiology. ACR appropriateness criteria: acute pyelonephritis. Reston (VA): The College; 1995. Available: www.acr.org/SecondaryMain MenuCategories/quality_safety/app_criteria/pdf/ExpertPanelonUrologicImaging/A cutePyelonephritisDoc3.aspx (accessed 2009 June 29) 